\title{
Check-list das samambaias e licófitas do estado de Mato Grosso do Sul, Brasil
}

\author{
Carlos Rodrigo Lehn', Elton Luis Monteiro de Assis² \& Alexandre Salino ${ }^{3}$
}

\begin{abstract}
'Instituto Federal Farroupilha, Campus Panambi, Rua Erechim 860, Bairro Planalto, CEP 98280-000. Panambi, RS, Brasil. crlehn@gmail.com 2Jardim Botânico do Rio de Janeiro, Rua Pacheco Leão, 915, Bairro Horto, CEP 22460-036, Rio de Janeiro, RJ, Brasil. ${ }^{3}$ Universidade Federal de Minas Gerais, Instituto de Ciências Biológicas, Departamento de Botânica, Av. Antônio Carlos 6627, Pampulha, CEP 31270-901, Belo Horizonte, MG, Brasil.
\end{abstract}

Recebido em 27.IX.2014

Aceito em 7.X.2015

DOI 10.21826/2446-8231201873s255

RESUMO - A presente listagem representa uma primeira compilação da diversidade das samambaias e licófitas para o estado de Mato Grosso do Sul. Encontramos registros de ocorrência de 207 táxons de samambaias (20\% das espécies de samambaias do Brasil) e 15 táxons de licófitas (12\% das espécies de licófitas do Brasil) distribuídos principalmente pelas regiões montanhosas do Maciço do Urucum na região oeste do estado. Esses dados são preliminares e evidenciam a necessidade de ampliação de estudos, visando `a redução de lacunas de coletas existentes e assim proporcionar um melhor conhecimento acerca da diversidade, ecologia e distribuição desses grupos de plantas no estado.

Palavras-chave: bioma, diversidade, Pteridófitas, Monilófitas, táxons

\begin{abstract}
Check-list of ferns and lycophytes of Mato Grosso do Sul state, Brazil. This checklist is the first compilation of ferns and lycophytes of Mato Grosso do Sul state, central-western, Brazil. We found occurrence records of 207 taxa of ferns ( $20 \%$ of the total number of Brazilian species) and 15 taxa of lycophytes ( $12 \%$ of the total number of Brazilian species), which were mainly distributed in the Urucum plateau, in the western region of the state. These data are preliminary and highlight the importance for further studies, which should aim to fill the gaps in herbarium collections, and thus provide a better understanding on the diversity, distribution, and ecology of these groups in the state.
\end{abstract}

Keywords: biome, diversity, ferns, Monilophytes, taxa

\section{INTRODUÇÃO}

As samambaias e licófitas se caracterizam principalmente pela reprodução e dispersão por meio de esporos e ciclo de vida com alternância de gerações independentes, sendo a gametofítica efêmera e a esporofítica duradoura com a morfologia e anatomia mais complexas. Recentemente, como resultado de estudos de filogenia molecular e morfologia, as plantas vasculares sem sementes foram agrupadas em dois grupos monofiléticos denominados de monilófitas e licófitas (Pryer et al. 2001). O primeiro compreende quatro classes (Psilotopsida, Equisetopsida, Marattiopsida e Polypodiopsida), 11 ordens (Ophioglossales, Psilotales, Equisetales, Marattiales, Osmundales, Hymenophyllales, Gleicheniales, Schizaeales, Salviniales, Cyatheales e Polypodiales) e 37 famílias, enquanto que o segundo é formado por Isoetaceae, Lycopodiaceae e Selaginellaceae (Smith et. al. 2006, 2008).

Atualmente, estima-se que a diversidade mundial de espécies de samambaias e licófitas seja de aproximadamente 12.000 espécies (PPG I 2016). Na América do Sul os dois grupos somam um total estimado em mais de 3.500 espécies (Moran 2008). No Brasil as licófitas estão representadas por 142 táxons e as samambaias por 1.111 (Prado et al. 2015).

No Brasil, samambaias e licófitas são encontradas em todos os domínios fitogeográficos, principalmente na
Floresta Atlântica que abriga cerca de $70 \%$ das espécies reconhecidas para a flora brasileira (Salino \& Almeida 2009), e que constitui um dos principais centros de diversidade e endemismo para esses grupos (Tryon \& Tryon 1982). As samambaias e licófitas se estabelecem em diversos ambientes desde as regiões úmidas e montanhosas do sul e sudeste até as mais secas da Caatinga e Cerrado do Brasil Central e consequentemente os estados pertencentes a estas regiões apresentam um grande numero de coletas e esses grupos de plantas mais amplamente estudados.

Em relação ao estado de Mato Grosso do Sul, estudos específicos para samambaias e licófitas ainda são bastante insipientes (Assis \& Labiak 2009a). As coletas mais antigas realizadas no estado remontam ao início do século XX, realizadas pela expedição da Comissão Rondon entre os anos de 1911-1913, chefiadas pelos botânicos naturalistas Frederich Carlos Hoehne e João Geraldo Kuhlmann, que catalogaram plantas coletadas durante essa expedição, dentre elas samambaias e licófitas (Hoehne 1951).

Mais recentemente, Pott \& Pott (2000) realizaram um estudo sobre as plantas aquáticas do Pantanal, onde fizeram considerações para 13 espécies de samambaias de hábito aquático ou palustre. Estudos mais específicos e sistematizados para os dois grupos em questão foram realizados por Assis (2007), Assis \& Labiak (2009a, 2009b) e Ponce et. al (2008) com foco nas regiões montanhosas da 
borda oeste do Pantanal e ainda por Lehn \& Assis (2013) ao inventariar as samambaias e licófitas ocorrentes em uma mata de galeria no interior do município de Aquidauana. Lehn et al. (2015) reportaram a primeira ocorrência de Elaphoglossum discolor (Kuhn) C.Chr para o Mato Grosso do Sul, uma espécie com distribuição amazônica.

Observa-se dessa maneira uma clara deficiência de estudos para esses grupos de plantas e de pesquisadores envolvidos em trabalhos dessa natureza no estado. Portanto, este "checklist" apresenta uma primeira abordagem sobre a diversidade e distribuição de samambaias e licófitas no estado de Mato Grosso do Sul.

\section{Principais Lacunas do Conhecimento}

Atualmente não existe nenhum grupo de pesquisa formalizado trabalhando com samambaias e licófitas no estado de Mato Grosso do Sul, o que se deve principalmente a falta de pesquisadores específicos vinculados às principais instituições de ensino superior do estado. Trabalhos de cooperação têm sido realizados ao longo dos últimos anos, envolvendo principalmente estudantes de pós-graduação e pesquisadores de outras regiões do Brasil.

O quantitativo de coletas envolvendo samambaias e licófitas observado para Mato Grosso do Sul ainda é muito pequeno. Cerca de $50 \%$ das espécies conhecidas para o estado ocorrem na região conhecida como Borda Oeste do Pantanal, onde coletas sistemáticas foram realizadas por mais de uma década e cujos resultados são apresentados em Assis (2007). Estima-se que estejam depositadas nos principais herbários do Brasil, pouco mais de 2.200 exemplares de samambaias e licófitas coletados em Mato Grosso do Sul.

A exceção da Borda Oeste do Pantanal, a maior parte das coletas envolvendo samambaias e licófitas foi realizada ao longo das principais rodovias do estado (Fig. 1), sendo possível observar extensas lacunas amostrais nas porções centro leste, centro norte, planície pantaneira e sudoeste. $\mathrm{O}$ incremento do esforço amostral, não exclusivamente, mas direcionado especialmente para estas áreas, contribuirá sobremaneira para o melhor conhecimento acerca da flora de samambaias e licófitas ocorrentes em Mato Grosso do Sul.

\section{Principais Acervos}

O maior acervo de samambaias e licófitas de Mato Grosso do Sul é o do Herbário COR da Universidade Federal de Mato Grosso do Sul/Campus do Pantanal, onde estão depositadas aproximadamente 1.400 exsicatas de samambaias e licófitas. No Herbário CGMS da Universidade Federal de Mato Grosso do Sul/Campus Campo Grande

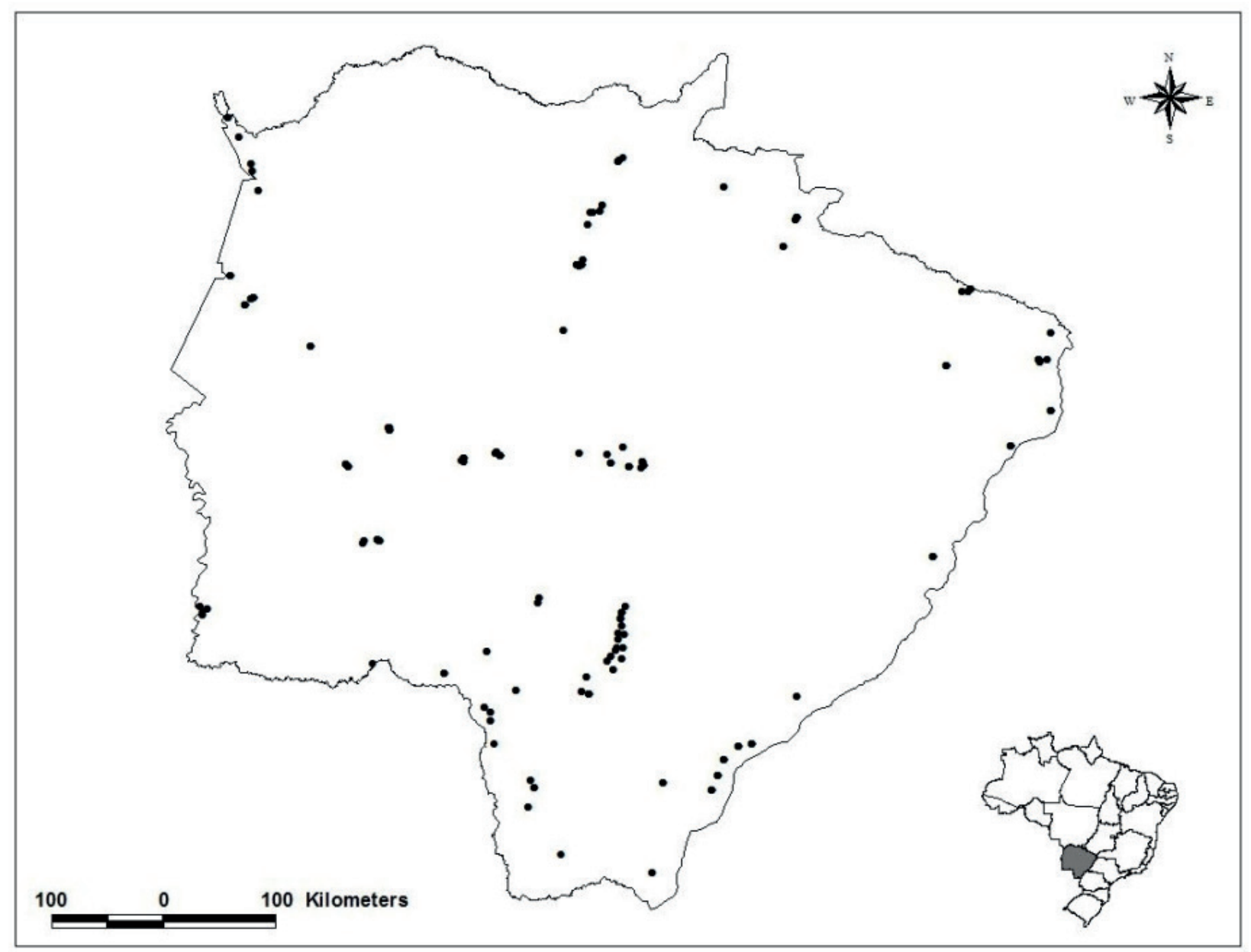

Fig. 1. Áreas com coletas de samambaias e licófitas no estado de Mato Grosso do Sul. 
estão depositadas pouco mais de 400 exsicatas. Os herbários MBM, SJRP e UPCB também reúnem uma quantidade significativa de amostras coletadas em Mato Grosso do Sul.

\section{Perspectivas de Pesquisas em Samambaias e Licófitas para os próximos 10 anos}

É importante que sejam definidas estratégias, diretrizes e metas para que haja um aumento no conhecimento acerca da flora de samambaias e licófitas ocorrente em Mato Grosso do Sul. Sugere-se que as seguintes estratégias sejam implementadas: a) contratação de taxonomistas visando a formação de grupos de pesquisas em samambaias e licófitas; b) estruturar um plano de coleta no estado visando cobrir as lacunas de amostragem e consequentemente enriquecer as coleções de samambaias e licófitas dos herbários; c) estabelecimento de parcerias com pesquisadores especialistas vinculados à instituições nacionais e estrangeiras, visando acelerar a identificação de espécies ocorrentes no estado; d) divulgar os resultados obtidos.

O investimento em infraestrutura para aperfeiçoamento dos laboratórios e herbários também é de fundamental importância para o melhor conhecimento da flora de Mato Grosso do Sul como um todo. Com o aumento do esforço amostral, será possível indicar áreas prioritárias para a conservação de samambaias e licófitas em Mato Grosso do Sul, bem como fornecer subsídios para a indicação de espécies que possam vir a integrar a lista vermelha da flora ameaçada do estado.

\section{MATERIAL E MÉTODOS}

A elaboração da presente listagem foi baseada em coleções de herbários do estado de Mato Grasso do Sul e do Brasil, principalmente do Herbário COR da Universidade Federal de Mato Grosso do Sul/Campus do Pantanal que constitui o maior acervo de samambaias e licófitas do estado e que serviu como base para citação dos espécimes testemunhos ("vouchers"). Foram analisados também materiais depositados nos herbários CGMS, CPAP, DDMS, BHCB, HSJRP, MBM, MO, RB, SPF e UPCB totalizando cerca de 2.000 exsicatas.

Para a ocorrência dos táxons no estado foram feitas consultas, além das coleções de herbários, ao "Lista da Flora do Brasil", artigos de floras regionais, revisões taxonômicas e livros. A partir dessas informações também foi confeccionado um mapa de distribuição, através do programa ArcMap 8.3 (Esri 1999-2002). As famílias dos táxons de samambaias estão apresentadas de acordo com o sistema de classificação proposto por Smith et al. (2006), e os de licófitas seguem Holub (1983), Haines (2003) e Øllgaard (2012) para a classificação de famílias e gêneros. Os nomes dos táxons em que não foi possível a análise do material são citados a fonte em que aparece como tendo ocorrência no estado ou ainda a "Lista de espécies da flora do Brasil" do Instituto de Pesquisas do Jardim Botânico do Rio de Janeiro. A circunscrição das famílias está de acordo com PPG I (2016). A abreviação do nome de autores segue
Pichi-Sermolli (1996).

\section{RESULTADOS E DISCUSSÃO}

O estado de Mato Grosso do Sul apresenta uma flora de samambaias e licófitas bastante diversificada com a ocorrência de 222 táxons o que corresponde a aproximadamente $20 \%$ do total ocorrentes no Brasil. Em relação às licófitas as espécies estão distribuídas entre as famílias Isoetaceae (duas spp.), Lycopodiaceae (sete spp.), e Selaginellaceae (seis spp.). Dentre as samambaias destacamse as famílias com o maior número de espécie: Pteridaceae (50 spp.), Polypodiaceae (34 spp.), Thelypteridaceae (23 spp.), Dryopteridaceae (18 spp.) e Aspleniaceae (16 spp.). A diversidade de espécies das demais famílias ocorrentes no estado está demonstrada na Figura 2

A região Centro-Oeste apresenta cerca de 394 espécies de samambaias e licófitas (Prado \& Sylvestre 2015), e aproximadamente $65 \%$ ocorrem em Mato Grosso do Sul. Embora não seja um dos mais diversos nesses grupos de plantas no Brasil como o são Minas Gerais (687 espécies - Salino \& Almeida 2009), e São Paulo (573 espécies - Prado \& Hirai 2011), o estado possui ambientes favoráveis ao estabelecimento dessas plantas como florestas estacionais semideciduais e deciduais, Cerrado sensu lato, campos de altitude e rupestres, matas de galeria e regiões chaquenhas. Entretanto, as espécies ocorrentes no estado são de ampla distribuição geográfica e até o momento apenas uma é considerada endêmica da região, Cheilanthes pantanalensis E.L.M.Assis et al., recentemente descrita para as áreas de cerrado da Serra do Amolar e com registro também para o Maciço do Urucum. Ainda assim não se descarta a possibilidade de sua ocorrência em regiões bolivianas próximas a localidade de ocorrência dessa espécie (Ponce et al. 2008). Dessa forma, a diversidade de samambaias conhecida para o estado até o presente momento é de 207 espécies, distribuídas em 67 gêneros e 24 famílias. Já as licófitas, estão representadas por 15 espécies, distribuídas em seis gêneros e três famílias (Quadro 1).

No estado, o Pantanal é o Bioma que apresenta maior diversidade, principalmente a região do Maciço do Urucum que coincide com o ponto mais alto de Mato Grosso do Sul, cerca de $1065 \mathrm{~m}$ (Silva 2000), e abriga aproximadamente $50 \%$ das espécies registradas até o momento para Mato Grosso do Sul. A maioria das espécies de samambaias e licófitas encontradas no estado cresce em ambientes úmidos das Florestas Estacionais Semideciduais e Matas de Galeria como representantes de Aspleniaceae, Cyatheaceae, Hymenophyllaceae e Polypodiaceae. Por outro lado, a minoria das espécies ocupa ambientes mais xéricos como o Cerrado e as matas secas, onde crescem espécies de Anemiaceae, Pteridaceae e Selaginellaceae ou ainda, podem ser encontradas nas áreas alagáveis da planície pantaneira como as Isoetaceae, Marsileaceae e Salviniaceae.

Estima-se que no estado devam ocorrer em torno de 300 espécies de samambaias e licófitas, tendo em vista que nesta primeira listagem foram acrescentadas 86 espécies que não constam no Catálogo de plantas e fungos do Brasil, 


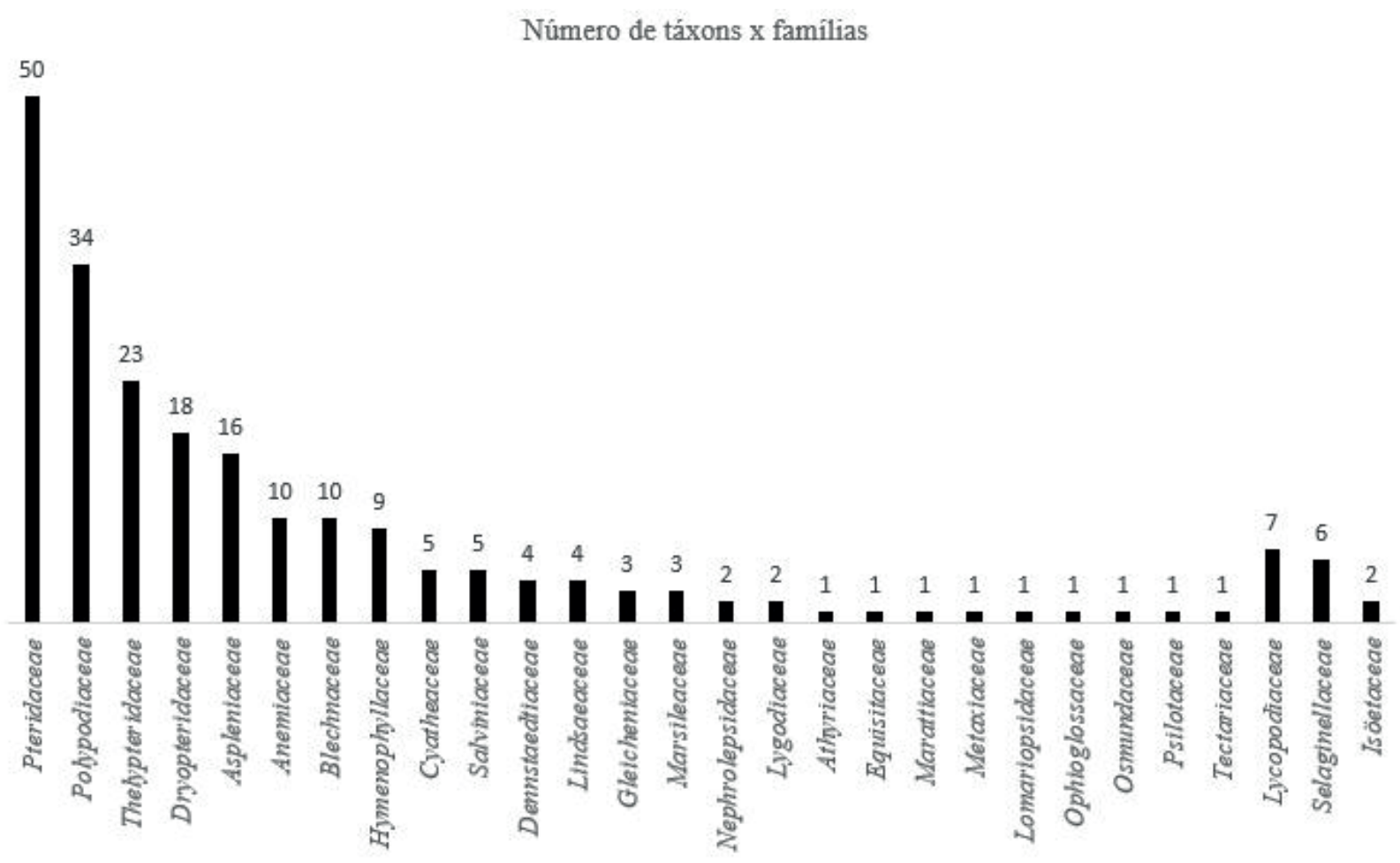

Fig. 2. Diversidade (número de táxons) das famílias de samambaias e licófitas em Mato Grosso do Sul.

Quadro 1. Lista de táxons de samambaias e licófitas do estado de Mato Grosso do Sul com os respectivos Vouchers ou referência de citação.

\begin{tabular}{|c|c|c|c|c|}
\hline Grupo & Família & Táxon & Voucher & Herbário \\
\hline \multirow[t]{15}{*}{ Licófitas } & Isoetaceae & Isoetes panamensis Maxon \& C.V.Morton & V.J. Pott et al. 11018 & CGMS \\
\hline & & I. pedersenii H.P.Fuchs ex E.I.Meza \& Macluf & $\begin{array}{l}\text { V.J. Pott \& S. N. Moreira } \\
10921\end{array}$ & CGMS \\
\hline & \multirow[t]{7}{*}{ Lycopodiaceae } & $\begin{array}{l}\text { Palhinhaea camporum (B.Øllg. \& P.G.Windisch) } \\
\text { Holub }\end{array}$ & E. Assis et al. 396 & COR, UPCB \\
\hline & & P. cernua (L.) Franco \& Vasc. & E. Assis et al. 26 & $\mathrm{BHCB}, \mathrm{COR}$ \\
\hline & & Phlegmariurus mandiocanus (Raddi) B.Øllg. & E. Assis \& D. Rodriguez 08 & $\mathrm{BHCB}, \mathrm{COR}$ \\
\hline & & P. reflexus (Lam.) B.Øllg. & E. Assis et al. 48 & $\mathrm{BHCB}, \mathrm{COR}$ \\
\hline & & Lycopodiella longipes (Grev. \& Hooker) Holub & W. G. Garcia 13747 & UEC \\
\hline & & Pseudolycopodiella carnosa (Silveira) Holub & $\begin{array}{l}\text { Citado em Lista da Flora } \\
\text { do Brasil }\end{array}$ & \\
\hline & & P. caroliniana (L.) Holub & E. Assis et al. 397 & COR, UPCB \\
\hline & \multirow[t]{6}{*}{ Selaginellaceae } & Selaginella chiquitana M. Kessler et al. & E. Assis 261 & $\mathrm{BHCB}, \mathrm{COR}$ \\
\hline & & S. convoluta (Arn.) Spring & E. Assis \& I.H. Ishii 238 & BHCB, COR, UPCB \\
\hline & & S. erythropus (Mart.) Spring & E. Assis et al. 409 & COR, UPCB \\
\hline & & S. marginata (Humb. \& Bonpl. ex Willd.) Spring & E. Assis et al. 52 & $\mathrm{BHCB}, \mathrm{COR}$ \\
\hline & & S. sellowii Hieron. & E. Assis 108 & $\mathrm{BHCB}, \mathrm{COR}$ \\
\hline & & S. sulcata (Desv. ex Poir.) Spring & E. Assis 368 & COR \\
\hline \multirow[t]{10}{*}{ Monilófitas } & \multirow[t]{10}{*}{ Anemiaceae } & Anemia australis (Mickel) M. Kessler \& A.R. Sm & E. Assis 130 & COR, UPCB \\
\hline & & Anemia buniifolia (Gardner.) T. Moore & V.J. Pott 10232 & CGMS \\
\hline & & A. ferruginea Humb. \& Bonpl. ex Kunth & Pietrobom-Silva et al. 2488 & SJRP \\
\hline & & A. herzogii Rosenst. & E. Assis 142 & COR, BHCB, UPCB \\
\hline & & A. phyllitidis (L.) Sw. & E. Assis 92 & $\mathrm{COR}$ \\
\hline & & A. pinnata Sehnem & $\begin{array}{l}\text { Damasceno Jr. \& Bortolo- } \\
\text { tto } 1047\end{array}$ & COR, UPCB \\
\hline & & A. raddiana Link & R. Betoni 106 & $\mathrm{BHCB}$ \\
\hline & & A. smithii Brade & C. R. Lehn 1075 & CGMS \\
\hline & & A. tomentosa var. anthriscifolia (Schrad.) Mickel & $\begin{array}{l}\text { E. Assis \& Damasceno } \\
\text { Jr. } 110\end{array}$ & COR \\
\hline & & A. trichorhiza Gardner & E. Assis 268 & COR, BHCB, UPCB \\
\hline
\end{tabular}


Quadro 1. Cont.

\begin{tabular}{|c|c|c|c|c|}
\hline Grupo & Família & Táxon & Voucher & Herbário \\
\hline & \multirow[t]{16}{*}{ Aspleniaceae } & Asplenium abscissum Willd. & E.P. Arteman 39 & CGMS \\
\hline & & A. auriculatum $\mathrm{Sw}$. & L.P. Clemente 82 & CGMS \\
\hline & & A. auritum $\mathrm{Sw}$. & E. Assis \& D. Rodriguez 57 & $\mathrm{BHCB}, \mathrm{COR}, \mathrm{UPCB}$ \\
\hline & & A. balansae (Baker) Sylvestre & R. Callejas 1920 & $\mathrm{RB}$ \\
\hline & & A. cristatum Lam. & L. P. Clemente 06 & CGMS \\
\hline & & A. depauperatum Fée & R. Forsa et al. 824 & SPF \\
\hline & & A. formosum Willd. & E. Assis et al. 39 & $\mathrm{BHCB}, \mathrm{COR}$ \\
\hline & & A. inaequilaterale Willd. & F. Firetti 43 & SJRP \\
\hline & & A. otites Link & E. Assis \& M.C. Brandão 42 & COR \\
\hline & & A. poloense Rosenst. & Sem coletor & SJRP-15086 \\
\hline & & A. praermosum $\mathrm{Sw}$. & E. Assis et al. 968 & COR \\
\hline & & A. radicans $\mathrm{L}$. & Pietrobom-Silva 1629 & MBM, SJRP \\
\hline & & A. salicifolium $\mathrm{L}$. & F.R. Nonato 258 & $\mathrm{RB}$ \\
\hline & & A. serra Langsd. \& Fisch. & N.R. Lenhard 27 & UPCB \\
\hline & & A. serratum $\mathrm{L}$. & Pietrobm-Silva 1300 & CGMS \\
\hline & & A. stuebelianum Hieron. & Damasceno Jr. et al. 3624 & $\mathrm{COR}$ \\
\hline & Athyriaceae & Diplazium cristatum (Desr.) Alston & E. Assis \& I.H. Ishii 139 & $\mathrm{COR}$ \\
\hline & \multirow[t]{10}{*}{ Blechnaceae } & Blechnum asplenioides $\mathrm{Sw}$. & C.E. Rodrigues Jr. 663 & SJRP \\
\hline & & B. austrobrasilianum de la Sota & E. Assis 271 & COR, UPCB \\
\hline & & B. laevigatum $\mathrm{Sw}$. & E. Assis 306 & COR, UPCB \\
\hline & & B. lanceola Sw. & C. R. Lehn 1050 & CGMS \\
\hline & & B. occidentale $\mathrm{L}$. & E. Assis et al. 30 & $\mathrm{BHCB}, \mathrm{COR}$ \\
\hline & & B. polypodioides Raddi & E. Assis et al. 265 & $\mathrm{BHCB}, \mathrm{COR}$ \\
\hline & & $\begin{array}{l}\text { Lomariocycas schomburgkii (Klotzsch) Gasper } \\
\text { \& A.R.Sm. }\end{array}$ & I. A. Carneiro et al. 71 & DDMS \\
\hline & & $\begin{array}{l}\text { Neoblechnum brasiliense (Desv.) Gasper \& } \\
\text { V.A.O.Dittrich }\end{array}$ & C.R. Lehn 1078 & CGMS \\
\hline & & Telmatoblechnum serrulatum (Rich.) Perrie et al. & Pietrobom-Silva 1126a & SJRP \\
\hline & & Salpichlaena volubilis (Kaulf.) J. Sm. & Pietrobom-Silva 1295 & SJRP \\
\hline & Cyatheaceae & Alsophila cuspidata (Kunze) D. S. Conant & E. Assis et al. 954 & COR \\
\hline & \multirow[t]{4}{*}{ Cyatheaceae } & Cyathea atrovirens (Langsd. \& Fisch.) Domin & $\begin{array}{l}\text { N. R. Lenhard \& Z. V. } \\
\text { Pereira } 56\end{array}$ & DDMS \\
\hline & & C. delgadii Sternb. & E. Assis 274 & $\mathrm{COR}$ \\
\hline & & C. microdonta (Desv.) Domin & E. Assis \& I.H. Ishii 76 & $\mathrm{BHCB}, \mathrm{COR}$ \\
\hline & & C. pungens (Willd.) Domin & I.A. Carneiro et. al. 54 & DDMS \\
\hline & \multirow[t]{4}{*}{ Dennstaedtiaceae } & Dennstaedtia cicutaria (Sw.) T. Moore & C.E. Rodrigues Jr. 725 & SJRP \\
\hline & & D. globulifera (Poir.) Hieron. & E. Assis et al. 94 & COR, UPCB \\
\hline & & D. mathewsii (Hook.) Hieron & E. Assis et al. 217 & $\mathrm{BHCB}, \mathrm{COR}, \mathrm{UPCB}$ \\
\hline & & Pteridium arachnoideum (Kaulf.) Maxon & E. Assis et al. 75 & $\mathrm{BHCB}, \mathrm{COR}, \mathrm{UPCB}$ \\
\hline & \multirow[t]{16}{*}{ Dryopteridaceae } & Bolbitis serratifolia Schott & E. Assis et al. 37 & $\mathrm{BHCB}, \mathrm{COR}$ \\
\hline & & Ctenitis submarginalis (Langsd. \& Fisch.) Ching & E. Assis \& D. Rodriguez 59 & $\mathrm{BHCB}, \mathrm{COR}$ \\
\hline & & $\begin{array}{l}\text { Cyclodium meniscioides (Wildd.) C.Presl var. } \\
\text { meniscioides }\end{array}$ & G.A. Damasceno Jr. 3030 & $\mathrm{COR}$ \\
\hline & & Didymochlaena truncatula (Sw.) J. Sm. & C.R. Lehn 1201 & CGMS \\
\hline & & Elaphoglossum discolor (Kuhn) C.Chr. & C.R. Lehn 1203 & SP \\
\hline & & E. horridulum (Kaulf.) J. Sm. & S.M.B. Silva 22 & UPCB \\
\hline & & E. lingua (C.Presl) Brack. & $\begin{array}{l}\text { Citado em Lista da Flora } \\
\text { do Brasil }\end{array}$ & \\
\hline & & E. luridum (Fée) Christ & $\begin{array}{l}\text { Citado em Lista da Flora } \\
\text { do Brasil }\end{array}$ & \\
\hline & & E. pachydermum (Fée) T.Moore & C.R. Lehn 1088 & CGMS \\
\hline & & E. piloselloides (C.Presl) T.Moore & C.R. Lehn 1055 & CGMS \\
\hline & & E. tectum (Humb. \& Bonpl. ex Willd.) T. Moore & $\begin{array}{l}\text { O.S. Ribas \& L.B.S. } \\
\text { Pereira } 2385\end{array}$ & MBM \\
\hline & & Lastreopsis effusa (Sw.) Tindale & I.A. Carneiro et al. 61 & CGMS \\
\hline & & Mickelia nicotianifolia (Sw.) R.C.Moran et al. & C.R. Lehn 1072 & CGMS \\
\hline & & Polybotrya caudata Kunze & M. Lucca Jr. 100 & SJRP \\
\hline & & P. fractiserialis (Baker) J. Sm. & E. Assis et al. 38 & $\mathrm{BHCB}, \mathrm{COR}$ \\
\hline & & P. goyazensis Brade & E. Assis \& D. Rodriguez. 77 & $\mathrm{BHCB}, \mathrm{COR}$ \\
\hline
\end{tabular}


Quadro 1. Cont.

\begin{tabular}{|c|c|c|c|c|}
\hline Grupo & Família & Táxon & Voucher & Herbário \\
\hline & & Polystichum platyphyllum (Willd.) C.Presl & F. Firetti & HSJRP-16397 \\
\hline & & Rumohra adiantiformis (Forst.) Ching & E. Assis \& Rodriguez 59 & $\mathrm{BHCB}, \mathrm{COR}$ \\
\hline & Equisetaceae & Equisetum giganteum L. & S.A. Cunha 271 & CGMS \\
\hline & Gleicheniaceae & Gleichenella pectinata (Willd.) Ching & $\begin{array}{l}\text { Citado em Lista da Flora } \\
\text { do Brasil }\end{array}$ & \\
\hline & & Dicranopteris flexuosa (Schrad.) Underw. & E. Assis et al. 27 & $\mathrm{BHCB}, \mathrm{COR}$ \\
\hline & & Sticherus lanuginosus (Fée) Nakai & E. Assis et al. 580 & COR, UPCB \\
\hline & Hymenophyllaceae & Abrodictyum rigidum (Sw.) Ebihara \& Dubuisson & Pietrobom-Silva 1269 & SJRP \\
\hline & & Didymoglossum hymenoides (Hedw.) Desv. & Souza et al. 02 & CGMS \\
\hline & & D. kraussii (Hook. \& Grev.) C.Presl & Pietrobom-Silva 1070 & SJRP \\
\hline & & Hymenophyllum polyanthos (Sw.) Sw. & F.R. Nonato 264a & SJRP \\
\hline & & Trichomanes cristatum Kaulf. & Clemente et al. 29 & DDMS \\
\hline & & T. hostmannianum (Klotzsch) Kunze & Pietrobom-Silva 1168 & SJRP \\
\hline & & T. pilosum Raddi & E. Assis 270 & $\mathrm{BHCB}, \mathrm{COR}, \mathrm{UPCB}$ \\
\hline & & T. pinnatum Hedw. & M. R. Pietrobom-Silva 867 & HSJRP \\
\hline & & T. polypodioides Raddi & E. Assis et al. 955 & COR \\
\hline & Lindsaeaceae & Lindsaea divaricata Klotzsch & I.A. Carneiro 41 & DDMS \\
\hline & & L. lancea (L.) Bedd & C.R. Lehn 1070 & CGMS \\
\hline & & L. quadrangularis Raddi & N.R. Lenhard et al. 126 & DDMS \\
\hline & & L. stricta (Sw.) Dryand var. stricta & E. Assis et al. 392 & COR, UPCB \\
\hline & Lomariopsidaceae & Lomariopsis nigropaleata Holttum & E. Assis et al. 251 & $\mathrm{BHCB}, \mathrm{COR}$ \\
\hline & Lygodiaceae & Lygodium venustum $\mathrm{Sw}$ & E. Assis et al. 31 & $\mathrm{BHCB}, \mathrm{COR}$ \\
\hline & & L. volubile Sw. & A. Amaral Jr. 368 & CGMS \\
\hline & Marattiaceae & Danaea nodosa (L.) Sm. & C.R. Lehn 1043 & CGMS \\
\hline & Marsileaceae & Marsilea crotophora D.M.Johnson & V. Pott et al. 1425 & CPAP \\
\hline & & M. deflexa A. Braun & V. Pott et al. 1445 & COR \\
\hline & & M. polycarpa Hook. \& Grev. & V. J. Pott et al. 1690 & CPAP \\
\hline & Metaxyaceae & Metaxya rostrata (Kunth) C.Presl & Berg et al. 19847 & MO \\
\hline & Nephrolepsidaceae & Nephrolepis biserrata (Sw.) Schott & Schio et al. & CGMS-06337 \\
\hline & & N. exaltata (L.) Schott & E. Assis et al. 107 & $\mathrm{BHCB}, \mathrm{UPCB}$ \\
\hline & Ophioglossaceae & Ophioglossum reticulatum $\mathrm{L}$. & V. J. Pott \& A. Rodrigues 3988 & CPAP \\
\hline & Osmundaceae & Osmunda regalis $\mathrm{L}$. & Lenhard et al. 46 & DDMS \\
\hline & Polypodiaceae & Campyloneurum acrocarpon Fée & U.M. Resende s.n. & CGMS \\
\hline & & C. austrobrasilianum (Alston) de la Sota & Pietrobom-Silva 879 & HSJRP \\
\hline & & C. brevifolium (Link) Link & Pietrobom-Silva & HSJRP-12567 \\
\hline & & C. centrobrasilianum Lellinger & E. Assis et al. 453 & COR, UPCB \\
\hline & & C. lapathifolium (Poir.) Ching & Resende et al. 1514 & COR \\
\hline & & C. nitidum (Kaulf.) C.Presl & Carneiro et al. 52 & DDMS \\
\hline & & C. phyllitidis (L.) C. Presl & E. Assis et al. 54 & $\mathrm{BHCB}, \mathrm{COR}$ \\
\hline & & C. repens (Aubl.) C.Presl & R. Betoni et al. 12 & DDMS \\
\hline & & Microgramma lindbergii (Mett.) de la Sota & M. R. Pietrobom-Silva 1215 & SJRP \\
\hline & & M. mortoniana de la Sota & E. Assis \& P. Swartsburd 528 & COR \\
\hline & & M. percussa (Cav.) de la Sota & $\begin{array}{l}\text { Citado em Lista da Flora } \\
\text { do Brasil }\end{array}$ & \\
\hline & & M. persicariifolia (Schrad.) C.Presl & Lenhard et al. 09 & DDMS \\
\hline & & M. squamulosa (Kaulf.) de la Sota & Pietrobom-Silva 3526 & SJRP \\
\hline & & M. vacciniifolia (Langsd. \& Fisch.) Copel. & E. Assis et al. 56 & $\mathrm{BHCB}, \mathrm{COR}$ \\
\hline & & Niphidium crassifolium (L.) Lellinger & $\begin{array}{l}\text { Citado em Lista da Flora } \\
\text { do Brasil }\end{array}$ & \\
\hline & & Pecluma dispersa (A.M. Evans) M.G. Price & E. Assis et al. 455 & COR, UPCB \\
\hline & & P. filicula (Kaulf.) M.G. Price & E. Assis et al. 89 & $\mathrm{BHCB}, \mathrm{COR}$ \\
\hline & & P. hoehnii (A. Samp.) Salino & F. C. Hoehne 3808 & $\mathrm{R}$ \\
\hline & & P. macedoi (Brade) M. Kessler \& A. R. Sm. & E. Assis et al. 40 & $\mathrm{BHCB}, \mathrm{COR}$ \\
\hline & & P. pectinatiformis (Lindm.) M. G. Price & Lenhard et al. 09 & DDMS \\
\hline & & $\begin{array}{l}\text { P. plumula (Humb. \& Bonpl. ex Willd.) M.G. } \\
\text { Price }\end{array}$ & E. Assis et al. 71 & COR \\
\hline & & P. robusta (Feé) M.Kessler \& A.R. Sm. & I.A. Carneiro et al. 35 & CGMS \\
\hline & & Phlebodium decumanum (Willd.) J.Sm. & E. Assis et al. 04 & $\mathrm{BHCB}, \mathrm{COR}$ \\
\hline & & P. pseudoaureum (Cav.) Lellinger & E. Assis et al. 48 & $\mathrm{BHCB}, \mathrm{COR}$ \\
\hline
\end{tabular}


Quadro 1. Cont.

\begin{tabular}{|c|c|c|c|c|}
\hline Grupo & Família & Táxon & Voucher & Herbário \\
\hline & \multirow{10}{*}{ Polypodiaceae } & Pleopeltis astrolepis (Liebm.) E. Fourn. & E. Assis et al. 24 & $\mathrm{BHCB}, \mathrm{COR}$ \\
\hline & & P. hirsutissima (Raddi) de la Sota & E. Assis \& Rodriguez 54 & $\mathrm{BHCB}, \mathrm{COR}$ \\
\hline & & P. minima (Bory) J. Prado \& R.Y.Hirai & E. Assis et al. 10 & COR \\
\hline & & P. pleopeltifolia (Raddi) Alston & Carneiro et al. 49 & DDMS \\
\hline & & P. polypodioides (L.) Andrews \& Windham & Pietrobom-Silva 789 & SJRP \\
\hline & & P. polypodioides var. burchellii (Baker) A.R.Sm. & $\begin{array}{l}\text { Citado em Lista da Flora } \\
\text { do Brasil }\end{array}$ & \\
\hline & & Serpocaulon fraxiniifolium (Jacq.) A. R. Sm. & Pietrobom-Silva 2448 & MBM \\
\hline & & S. latipes (Langsd. \& Fisch.) A. R. Sm. & E. Assis \& D. Rodriguez 47 & $\mathrm{BHCB}, \mathrm{COR}$ \\
\hline & & S. triseriale (Sw.) A.R.Sm. & Carneiro et al. 70 & DDMS \\
\hline & & S. vacillans (Link) A.R.Sm. & N.R. Lenhard 56 & DDMS \\
\hline & \multirow{20}{*}{$\begin{array}{l}\text { Psilotaceae } \\
\text { Pteridaceae }\end{array}$} & Psilotum nudum (L.) P.Beauv. & C.R. Lehn 1024 & CGMS \\
\hline & & Acrostichum danaeifolium Langsd. \& Fisch. & E. Assis et al. 404 & COR, UPCB \\
\hline & & Adiantopsis chlorophylla (Sw.) Fée & $\begin{array}{l}\text { G. Hatschbach et al. } \\
74461\end{array}$ & MBM \\
\hline & & A. senae (Baker) Schuettp. \& A. Davilla & C.E Rodrigues Jr. 645 & SJRP \\
\hline & & A. perfasciculata Sehnem & E. Assis et al. 374 & COR, UPCB \\
\hline & & A. radiata $(\mathrm{L}$.$) Fée$ & C.R. Lehn 1074 & CGMS \\
\hline & & A. flexuosa (Kunze) Link-Pérez \& Hinckey & E. Assis et al. 224 & COR, UPCB \\
\hline & & Adiantum abscissum Schrad. & E. Assis 147 & $\mathrm{BHCB}, \mathrm{COR}, \mathrm{UPCB}$ \\
\hline & & A. curvatum Kaulf. & $\begin{array}{l}\text { E.P. Arteman \& A.M. } \\
\text { Oliveira } 5\end{array}$ & BHCB, DDMS \\
\hline & & A. deflectens Mart. & E. Assis et al. 43 & $\mathrm{BHCB}, \mathrm{COR}$ \\
\hline & & A. delicatulum Mart. & G.G. Hatschbach 23769 & MBM \\
\hline & & A. diogoanum Glaziou ex Backer & Bueno et al. 78 & CPAP \\
\hline & & A. intermedium Sw. & Souza et al. 26824 & MO \\
\hline & & A. latifolium Lam. & E. Assis et al. 363 & COR, MBM, UPCB \\
\hline & & A. lorentzii Hieron. & G.G. Hatschbach 974 & MO \\
\hline & & A. mathewsianum Hook. & E. Assis et al. 61 & $\mathrm{BHCB}, \mathrm{COR}$ \\
\hline & & A. nudum A. R. Sm. & H.S. Irwin 17092 & $\mathrm{RB}$ \\
\hline & & A. obliquum Willd. & V.J. Pott 5659 & CGMS \\
\hline & & A. patens Willd. & E. Assis 147 & $\mathrm{COR}$ \\
\hline & & A. pectinatum Kunze ex Baker & Bento et al. 1498 & $\mathrm{COR}$ \\
\hline & \multirow[t]{20}{*}{ Pteridaceae } & A. phyllitidis J.Sm. & $\begin{array}{l}\text { Citado em Lista da Flora } \\
\text { do Brasil }\end{array}$ & \\
\hline & & A. platyphyllum $\mathrm{Sw}$. & $\begin{array}{l}\text { E.P. Arteman \& A.M. } \\
\text { Oliveira } 14\end{array}$ & BHCB, DDMS \\
\hline & & A. poiretii (Mett. ex Kuhn) Crabbe & G.A. Damasceno Jr. 1117 & $\mathrm{COR}$ \\
\hline & & A. pseudotinctum Hieron. & Matos et al. 1502 & $\mathrm{COR}$ \\
\hline & & A. serratodentatum Willd. & E. Assis et al. 97 & $\mathrm{COR}$ \\
\hline & & A. tetraphyllum Willd. & G.A. Damasceno Jr. 3037 & COR \\
\hline & & Ceratopteris pteridoides (Hook.) Hieron. & Bastos 04 & CPAP \\
\hline & & C. thalictroides (L.) Brongn. & $\begin{array}{l}\text { Citado em Lista da Flora } \\
\text { do Brasil }\end{array}$ & \\
\hline & & $\begin{array}{l}\text { Cheilanthes geraniifolia (Weath.) R.M.Tryon \& } \\
\text { A.F.Tryon }\end{array}$ & E. Assis et al. 492 & COR, SI, UPCB \\
\hline & & C. goyazensis (Taub.) Domin & E. Assis \& I. H. Ishii 267 & $\begin{array}{l}\text { BHCB, COR, SI, } \\
\text { UPCB }\end{array}$ \\
\hline & & C. hassleri (Weath.) Ponce & $\begin{array}{l}\text { G.A. Damasceno Jr. et } \\
\text { al. } 4196\end{array}$ & $\mathrm{COR}$ \\
\hline & & C. obducta Mett. ex Kuhn & $\begin{array}{l}\text { E. Assis \& G. A. Damas- } \\
\text { ceno Jr. } 280\end{array}$ & $\mathrm{BHCB}, \mathrm{COR}, \mathrm{SI}$ \\
\hline & & C. pantanalensis E.L.M.Assis et al. & E. Assis et al. 364 & COR, SI, SP, UPCB \\
\hline & & C. tweediana Hook. & E. Assis et al. 109 & $\mathrm{BHCB}, \mathrm{COR}$ \\
\hline & & Doryopteris collina (Raddi) J. Sm. & E. Assis 257 & $\mathrm{BHCB}, \mathrm{COR}, \mathrm{MBM}$ \\
\hline & & D. concolor (Langsd. \& Fisch.) J.Sm. & E. Assis 106 & $\mathrm{COR}$ \\
\hline & & D. lorentzii (Hieron.) Diels in Engl. \& Prantl & $\begin{array}{l}\text { E. Assis \& P. Schwarts- } \\
\text { burd } 569\end{array}$ & COR, UPCB \\
\hline & & D. nobilis (T.Moore) C.Chr. & Pereira et al., 14 & DDMS \\
\hline & & D. pentagona Pic.Serm. & Clemente et al. 19 & DDMS \\
\hline & & Hecistopteris pumila (Spreng.) J.Sm. & $\begin{array}{l}\text { Citado em Lista da Flora } \\
\text { do Brasil }\end{array}$ & \\
\hline
\end{tabular}


Quadro 1. Cont.

\begin{tabular}{|c|c|c|c|c|}
\hline Grupo & Família & Táxon & Voucher & Herbário \\
\hline & \multirow{11}{*}{ Pteridaceae } & Hemionitis tomentosa (Lam.) Raddi & C.R. Lehn 1073 & CGMS \\
\hline & & Lytoneuron lomariaceum (Klotzsch) J.C.Yesilyurt & U. M. Resende et al. 97 & CGMS \\
\hline & & L. ornithopus (Hook. \& Baker) J.C.Yesilyurt & G.G. Hatschbach 35995 & MO \\
\hline & & $\begin{array}{l}\text { Pityrogramma calomelanos (L.) Link var. calo- } \\
\text { melanos }\end{array}$ & E. Assis et al. 06 & $\mathrm{BHCB}, \mathrm{COR}$ \\
\hline & & P. trifoliata (L.) R.M.Tryon & C. R. Lehn 1030 & CGMS \\
\hline & & Pteris deflexa Link & S.M.B. Silva 37 & UPCB \\
\hline & & P. denticulata Sw. & G. Hatschbach 47233 & MBM \\
\hline & & P. plumula Desv. & E. Assis et al. 07 & $\mathrm{BHCB}, \mathrm{COR}$ \\
\hline & & P. propinqua J.Agardh & E. Assis 64 & $\mathrm{BHCB}, \mathrm{COR}$ \\
\hline & & $P$. vittata $\mathrm{L}$ & E. Assis 112 & $\mathrm{BHCB}, \mathrm{COR}$ \\
\hline & & Vittaria lineata (L.) Sm. & E. Assis et al. 200 & COR \\
\hline & \multirow[t]{2}{*}{ Salviniaceae } & Azolla caroliniana Willd. & Sanches et al. 40 & COR \\
\hline & & A. filiculoides Lam. & E. Assis 66 & $\mathrm{BHCB}, \mathrm{COR}, \mathrm{CPAP}$ \\
\hline & \multirow{3}{*}{$\begin{array}{l}\text { Salvinia auricula- } \\
\text { ta Aubl. }\end{array}$} & E. Assis et al. 121 & COR, MBM, UPCB & \\
\hline & & S. biloba Raddi & E. Assis et al. 328 & COR, UPCB \\
\hline & & $S$. minima Baker & E. Assis et al. 120 & COR, MBM, UPCB \\
\hline & Tectariaceae & $\begin{array}{l}\text { Tectaria incisa Cav. f. vivipara (Jenman) C.V. } \\
\text { Morton }\end{array}$ & E. Assis et al. 63 & $\mathrm{BHCB}, \mathrm{COR}$ \\
\hline & \multirow[t]{23}{*}{ Thelypteridaceae } & $\begin{array}{l}\text { Amauropelta heineri (C.Chr.) Salino \& T.E.Al- } \\
\text { meida }\end{array}$ & S.M.B. Silva 33 & $\mathrm{BHCB}$ \\
\hline & & A. mosenii (C.Chr.) Salino \& T.E.Almeida & M. Ravanelli s.n. & COR \\
\hline & & A. opposita (Vahl) Pic.Serm. & V.J. Pott 6420 & CGMS \\
\hline & & A. rivularioides (Fée) Salino \& T.E.Almeida & I.A. Carneiro 24 & DDMS \\
\hline & & A. saxicola (Sw.) C. F. Reed & V.J. Pott 6419 & CGMS \\
\hline & & Amblovenatum opulentum (Kaulf.) J.P.Roux & E. Assis 910 & COR \\
\hline & & $\begin{array}{l}\text { Christella conspersa (Schrad.) Á.Löve \& } \\
\text { D.Löve }\end{array}$ & E. Assis et al. 29 & $\mathrm{BHCB}, \mathrm{COR}$ \\
\hline & & C. dentata (Forssk.) Brownsey \& Jermy & E. Assis \& I. Ishii 09 & $\mathrm{BHCB}, \mathrm{COR}$ \\
\hline & & C. hispidula (Decne.) Holttum & E. Assis 76 & $\mathrm{BHCB}, \mathrm{COR}, \mathrm{CPAP}$ \\
\hline & & C. patens (Sw.) Pic.Serm. & R.M.B. Silva 05 & CGMS \\
\hline & & Cyclosorus interruptus (Willd.) H.Ito & E. Assis \& P. Swartsburd 556 & COR \\
\hline & & $\begin{array}{l}\text { Goniopteris jamesonii (Hook.) Salino \& } \\
\text { T.E.Almeida }\end{array}$ & S.M.B. Silva 02 & $\mathrm{BHCB}$ \\
\hline & & G. lugubris (Mett.) Brade & E. Assis \& A. Takahasi 556 & COR, UPCB, MBM \\
\hline & & G. scabra (C.Presl) Brade & I.A. Carneiro 12 & DDMS \\
\hline & & Macrothelypteris torresiana (Gaudich.) Ching & E. Assis \& I. H. Ishii 01 & $\mathrm{BHCB}, \mathrm{COR}$ \\
\hline & & Meniscium angustifolium Willd & C.R. Lehn 1090 & CGMS \\
\hline & & M. arborescens Humb. \& Bonpl. ex Willd. & G.A. Damasceno junior 3033 & COR \\
\hline & & M. longifolium Desv. & E. Assis et al. 124 & COR, UPCB \\
\hline & & $\begin{array}{l}\text { Meniscium maxonianum (A.R.Sm.) R.S.Fernan- } \\
\text { des \& Salino }\end{array}$ & Pietrobom-Silva 1149 & HSJRP \\
\hline & & M. serratum Cav. & C.R. Lehn 1032 & CGMS \\
\hline & & Thelypteris berroi (C.Chr.) C.F.Reed & Pietrobom-Silva 1336 & SJRP \\
\hline & & T. grandis var. kunzeana (Hook.) A.R.Sm. & E. Assis 195 & COR, UPCB, MBM \\
\hline & & Steiropteris leprieurii (Hook.) Pic.Serm. & R.M.B. Silva 06 & CGMS \\
\hline
\end{tabular}

que possui uma seção organizada por Prado \& Sylvestre (2015), para esses grupos de plantas. Pode-se verificar ainda a ocorrência de espécies restritas a Mato Grosso do Sul no território brasileiro como: Adiantopsis flexuosa (Kunze) Link-Pérez \& Hickey, Anemia herzogii Rosenst., Cheilanthes hassleri (Weath.) Ponce, Cheilanthes obducta Mett ex Kuhn, Cheilanthes tweediana Hook., Dennstaedtia mathewsii (Hook.) C.Chr., Isoetes pedersenii H.P. Fuchs ex E.I. Meza \& Macluf, Polybotrya fractiserialis (Baker) J. Sm. e Selaginella chiquitana M. Kessler et al. .

A região melhor estudada no estado corresponde a borda oeste do Pantanal onde se localiza o Herbário COR da Universidade Federal de Mato Grosso do Sul/CPAN que possuí um acervo de mais de 1.400 exsicatas de espécimes desses grupos de plantas com ocorrência no estado. Ainda assim, há uma enorme lacuna de coletas para o estado principalmente nas regiões noroeste e leste (Fig. 2).

A presente listagem, longe de ser conclusiva, reflete a necessidade de envolver as samambaias e licófitas em estudos florísticos, fitossociológicos e até moleculares, pois constituem elementos bastante diversos na flora brasileira e para Mato Grosso do Sul ainda não se tem um conhecimento 
real da diversidade destes grupos. A formação de recursos humanos constitui outra necessidade na região, alunos de graduação e pós-graduação que estudem esses grupos de plantas no estado, principalmente nas áreas onde a defasagem de coletas é maior. Esses estudos contribuirão sobremaneira para que a flora de samambaias e licófitas de Mato Grosso do Sul seja melhor conhecida, com grandes possibilidades não somente de registros novos bem como a descrição de espécies novas, além de compreender a ecologia, biogeografia e ampliar a distribuição de vários táxons desses grupos de plantas.

\section{AGRADECIMENTOS}

Os autores agradecem aos curadores dos herbários visitados e a Marcelo Leandro Bueno pelo auxílio na confecção do mapa das áreas coletadas.

\section{REFERÊNCIAS}

Assis, E. L. M. 2007. Pteridófitas da Borda Oeste do Pantanal sulmatogrossense, Brasil. Dissertação 200 f., Universidade Federal do Paraná, Curitiba.

Assis, E. L. M. \& Labiak, P. H. 2009a. Lycophyta da borda oeste do Pantanal, Mato Grosso do Sul, Brasil. Acta botanica brasilica 23(3): 703-712.

Assis, E. L. M. \& Labiak, P. H. 2009b. Polypodiaceae da borda oeste do Pantanal sul-matogrossense, Brasil. Revista Brasileira de Botânica 32(2): 233-247.

Esri 1999-2002. ARCMAPTM for Windows version 8.3. Redlands. Environmental System Research Institute. CDROM.

Haines, A. 2003. The Families Huperziaceae and Lycopodiaceae in New England. V.F. Thomas Co., Bowdoin. 100p.

Hoehne, F. C. 1951. Índice Bibliográfico e Numérico das Plantas Colhidas pela Comissão Rondon. Instituto de Botânica de São Paulo, São Paulo. 400 p.

Holub, J. 1983. Validation of generic names in Lycopodiaceae: with a description of a new genus Pseudolycopodiella. Folia Geobotanica Phytotaxonomica 18:439-442.

Lehn, C. R. \& Assis, E. L. M. 2013. Riqueza de samambaias e licófitas de uma mata de galeria na região central de Mato Grosso do Sul,
Brasil. Biotemas 26(1):7-15

Lehn, C. R.; Assis, E. L. M. \& Neves, D. M. 2015. Elaphoglossum discolor (Kuhn) C.Chr (Dryopteridaceae, Polypodiales, Monilophyta): first record for the state of Mato Grosso do Sul, Brazil. Check List 11: 1-3.

Moran, R. C. 2008. Diversity, biogeography and floristics. In Biology and Evolution of Ferns and Lycophytes (Ranker, T.A. \& Haufler, C.H., orgs.).Cambridge University Press, Cambridge, p. 367 -394

Øllgaard, B. 2012. Nomenclatural changes in Brazilian Lycopodiaceae. Rodriguésia 63(2): 479-482.

Pichi-Sermolli, R.E.G. 1996. Authors of scientific names in Pteridophyta. Kew: Royal Botanic Gardens, 78 p.

Ponce, M, M., Assis, E. L. M. \& Labiak, P. H. 2008. A new species and two new records of the fern genus Cheilanthes (Pteridaceae) from Southwestern Brazil. American Fern Journal 98(4): 202-207.

Pott, V. J. \& Pott, A. 2000. Plantas Aquáticas do Pantanal. Brasília: Empresa Brasileira de Pesquisa Agropecuária. 404p.

PPG I. 2016. A community-derived classification for extant lycophytes and ferns. Journal of Systematics and Evolution 54(6):563-603.

Prado, J. \& Hirai, R. 2011. Checklist das licófitas e samambaias do Estado de São Paulo, Brasil. Biota Neotropica 11(1): 161-190.

Prado, J. \& Sylvestre, L. 2015. Pteridófitas. In Lista de Espécies da Flora do Brasil. Jardim Botânico do Rio de Janeiro. Disponível em: http://floradobrasil.jbrj.gov.br/jabot/floradobrasil/FB128483. Acessado em 09.09.2015.

Prado, J. et al. 2015. Diversity of ferns and lycophytes in Brazil. Rodriguésia 66(4): 1-12.

Pryer, K.M., Schneider, H., Smith, A.R., Cranfill, R., Wolf, P.G., Hunt, J.S. \& Sipes, S.D. 2001. rbcL data reveal two monophyletic groups of filmy ferns (Filicopsida: Hymenophyllaceae). American Journal of Botany 88: 1118-1130.

Salino, A. \& Almeida, T. E. 2009. Pteridófitas. In Plantas da Floresta Atlântica (J. R. Stehmann, R. C. Forzza, A. Salino, M. Sobral, D. P. Costa \& L. H. Y. Kamino, L.H.Y., orgs). Jardim Botânico do Rio de Janeiro, Rio de Janeiro. p.19-25.

Silva, J. S. V. 2000. Zoneamento Ambiental da Borda Oeste do Pantanal: Maciço do Urucum e adjacências. Brasília: Empresa Brasileira de Pesquisa Agropecuária, $211 \mathrm{p}$

Smith, A. R., Kathleen, M. P., Schuettpelz, E., Korall, P., Schneider, H. \& Wolf, P. G. 2006. A classification for extant ferns. Taxon 55:705-731,

Smith, A. R., Pryer, K. M., Schuettpelz, E., Korall, P., Schneider, H. \& Wolf, P. G. 2008. Fern classification. In The Biology and Evolution of Ferns and Lycophytes (Ranker, T.A. \& Haufler, C.H., orgs.). Cambridge University Press, Cambridge, p. 417-467.

Tryon, R. M. \& Tryon, A. F. 1982. Ferns and allied plants with special reference to tropical America. New York: Springer, 857 p. 